\title{
Excited light isoscalar mesons from lattice QCD
}

\author{
Christopher E. Thomas* (for the Hadron Spectrum Collaboration) \\ Jefferson Laboratory, 12000 Jefferson Avenue, Newport News, VA 23606, USA \\ E-mail: thomasc@jlab.org
}

I report a recent lattice QCD calculation of an excited spectrum of light isoscalar mesons, something that has up to now proved challenging for lattice QCD. With novel techniques we extract an extensive spectrum with high statistical precision, including spin-four states and, for the first time, light isoscalars with exotic quantum numbers. In addition, the hidden flavour content of these mesons is determined, providing a window on annihilation dynamics in QCD. I comment on future prospects including applications to the study of resonances.

The XXIX International Symposium on Lattice Field Theory - Lattice 2011

July 10-16, 2011

Squaw Valley, Lake Tahoe, California

${ }^{*}$ Speaker. 
Over the last few years there has been a resurgence of interest in meson spectroscopy lead by a wealth of data emerging from various experiments which has in turn fuelled much theoretical work. Experimental interest in the light meson sector continues with BESIII, COMPASS, KLOE, upcoming GlueX and CLAS12 (part of Jefferson Lab's $12 \mathrm{GeV}$ upgrade), and the planned PANDA experiment. In particular, GlueX aims to perform a systematic investigation of the light meson spectrum in photoproduction and, notably, to produce mesons with exotic quantum numbers, such as $J^{P C}=0^{+-}, 1^{-+}$and $2^{+-}$. Such an exotic state signals physics beyond that of just a quarkantiquark pair, one possibility being a hybrid meson where the gluonic degrees of freedom play a nontrivial role.

An essential ingredient in testing QCD is the calculation of the spectrum of QCD and comparison against high quality experimental data. Lattice QCD provides a first-principles method for computing the spectrum and the calculation of the masses of the lowest-lying states has long been an important benchmark of such calculations. The Hadron Spectrum Collaboration has recently made significant progress in studying excited states using lattice QCD calculations, first testing techniques for extracting excited spectra [1] and radiative transition amplitudes $[2,3]$ with a quenched calculation in the charmonium sector, where there is a profusion of experimental data. These methods are now being applied to the light meson sector with an eventual aim of calculating excited and exotic meson photocouplings, relevant for, amongst other things, the GlueX experiment.

The first excited light meson spectra extracted in this programme of dynamical lattice QCD calculations, Refs. $[4,5]$, were of isovector $(I=1)$ mesons across all parity $(P)$ and chargeconjugation parity $(C)$ combinations. An important advance was the development of techniques which enable the continuum spin of mesons to be extracted in a reliable way at a single lattice spacing. The results include the most extensive excited spectrum ever obtained from a lattice QCD calculation, many states with exotic quantum numbers $\left(0^{+-}, 1^{-+}\right.$and $\left.2^{+-}\right)$and, for the first time in such a calculation, spin-four $\left(4^{++}, 4^{-+}, 4^{--}\right)$states. Another highlight is a non-exotic vector hybrid state, identified by its large overlap with an operator proportional to the gluonic field-strength tensor, suggesting that the gluonic field plays a non-trivial role in this state. A more model-dependent analysis of the results, identifying the lightest hybrid supermultiplet, has been performed lately [6]. These techniques are also beginning to be applied to the charmonium sector $[7,8]$.

More recently we have studied light isoscalar $(I=0)$ mesons [9]. Isoscalars, with no net flavour, can be constructed from quark-antiquark pairs of any flavour and even from states of pure glue. Studying the spectrum of isoscalars and the admixtures of the different flavour basis states in the physical states allows us to probe QCD annihilation dynamics. As described in Ref. [9], isoscalars are a challenging undertaking in lattice QCD for several reasons. Lattice gauge configurations with dynamical light and strange quarks are required so that the flavour mixing appears in a unitary way and the evaluation of disconnected correlator contributions is computationally expensive with signals typically decaying into noise at small Euclidean times. As described in that reference, significant progress has been made through novel computational techniques and the use of Graphical Processing Units (GPUs) to perform some parts of the calculations. 
An extensive spectrum was extracted [9] (at a single lattice spacing and volume, and with $m_{\pi} \sim 400 \mathrm{MeV}$ ) and suggests a phenomenology that is in quite good agreement with experiment. The pattern of $\eta-\eta^{\prime}$ observed in experiment is reproduced with a light-strange mixing angle, $42(1)^{\circ}$, in line with phenomenological determinations. In the vector channel the statistical precision is such that we determine the $\omega-\rho$ mass splitting to be $21(5) \mathrm{MeV}$. Another highlight is the first computation of a spectrum of isoscalar mesons with exotic $J^{P C}$; these appear at a comparable mass scale to the exotic isovectors. We observe that the majority of states we extract are close to ideally mixed with notable exceptions of $\eta$ and $\eta^{\prime}$, the axial vector states $\left(1^{++}\right)$, and the $1^{-+}$exotics.

As described elsewhere in these proceedings [10], these computations, combined with techniques for extracting energy-dependent phase shifts, lay the groundwork for using lattice QCD to investigate meson-meson scattering channels where quark annihilation contributions are required. This will enable us to study the $I=1 \pi \pi$ sector where the $\rho$ resonance is expected to appear as a rapidly rising phase shift. Some attempts in this direction have already been made [11-15] but these calculations only extract a limited number of phase shift points. A combination of techniques, including Distillation [16] and stochastic variants [17], and the consideration of multi-meson states at overall non-zero momentum [18], will enable us to map out many points on the phase shift curve. Future work will explore a range of scattering channels in various partial waves, with particular emphasis on the determination of hadronic resonances.

\section{Acknowledgments}

I thank Jo Dudek, Robert Edwards, Bálint Joó, Mike Peardon, David Richards and other colleagues in the Hadron Spectrum Collaboration. The work discussed here used Chroma [19] and QUDA $[20,21]$ on clusters at Jefferson Lab under the USQCD Initiative and the LQCD ARRA project. Gauge configurations were generated using resources awarded from the U.S. Department of Energy INCITE program at Oak Ridge National Lab, the NSF Teragrid at the Texas Advanced Computer Center and the Pittsburgh Supercomputer Center, as well as at Jefferson Lab.

Authored by Jefferson Science Associates, LLC under U.S. DOE Contract No. DE-AC0506OR23177. The U.S. Government retains a non-exclusive, paid-up, irrevocable, world-wide license to publish or reproduce this manuscript for U.S. Government purposes.

\section{References}

[1] J. J. Dudek, R. G. Edwards, N. Mathur, and D. G. Richards, Charmonium excited state spectrum in lattice QCD, Phys. Rev. D77 (2008) 034501, [arXiv:0707.4162].

[2] J. J. Dudek, R. G. Edwards, and D. G. Richards, Radiative transitions in charmonium from lattice QCD, Phys. Rev. D73 (2006) 074507, [hep-ph/ 0601137 ].

[3] J. J. Dudek, R. Edwards, and C. E. Thomas, Exotic and excited-state radiative transitions in charmonium from lattice QCD, Phys. Rev. D79 (2009) 094504, [arXiv: 0902 . 2241].

[4] J. J. Dudek, R. G. Edwards, M. J. Peardon, D. G. Richards, and C. E. Thomas, Highly excited and exotic meson spectrum from dynamical lattice QCD, Phys. Rev. Lett. 103 (2009) 262001, [arXiv:0909.0200]. 
[5] J. J. Dudek, R. G. Edwards, M. J. Peardon, D. G. Richards, and C. E. Thomas, Toward the excited meson spectrum of dynamical QCD, Phys. Rev. D82 (2010) 034508, [arXiv: 1004.4930 ].

[6] J. J. Dudek, The lightest hybrid meson supermultiplet in QCD, arXiv:1106.5515.

[7] L. Liu et. al., Charmonium spectrum from anisotropic lattices, , 2011 in these proceedings.

[8] S. Ryan et. al., Disconnected diagrams in charmonium physics, , 2011 in these proceedings.

[9] J. J. Dudek et. al., Isoscalar meson spectroscopy from lattice QCD, Phys. Rev. D83 (2011) 111502, [arXiv:1102.4299].

[10] D. Richards, Energy-dependent $i=2$ pi-pi scattering phase shift in lattice QCD, , 2011 in these proceedings.

[11] S. Aoki et. al. (CP-PACS), Lattice QCD Calculation of the $\rho$ Meson Decay Width, Phys. Rev. D76 (2007) 094506, [arXiv: 0708 .3705].

[12] M. Gockeler et. al. (QCDSF), Extracting the rho resonance from lattice QCD simulations at small quark masses, PoS LATTICE2008 (2008) 136, [arXiv: 0810 . 5337].

[13] X. Feng, K. Jansen, and D. B. Renner, Resonance Parameters of the rho-Meson from Lattice QCD, Phys. Rev. D83 (2011) 094505, [arXiv: 1011.5288 ].

[14] C. B. Lang, D. Mohler, S. Prelovsek, and M. Vidmar, Coupled channel analysis of the rho meson decay in lattice QCD, arXiv:1105.5636.

[15] T. P.-. S. Aoki et. al. (CS), $\rho$ Meson Decay in 2+1 Flavor Lattice QCD, arXiv: 1106.5365.

[16] M. Peardon et. al. (Hadron Spectrum), A novel quark-field creation operator construction for hadronic physics in lattice QCD, Phys. Rev. D80 (2009) 054506, [arXiv: 0905.2160 ].

[17] C. Morningstar et. al., Improved stochastic estimation of quark propagation with Laplacian Heaviside smearing in lattice QCD, Phys. Rev. D83 (2011) 114505, [arXiv:1104.3870].

[18] C. E. Thomas, R. G. Edwards, and J. J. Dudek, Helicity operators for mesons in flight on the lattice, arXiv:1107.1930. to be published in Phys. Rev. D.

[19] R. G. Edwards and B. Joo (SciDAC Collaboration), The chroma software system for lattice qcd, Nucl. Phys. B. Proc. Suppl. 140 (2005) 832, [hep-lat/ 0409003 ].

[20] M. A. Clark, R. Babich, K. Barros, R. C. Brower, and C. Rebbi, Solving Lattice QCD systems of equations using mixed precision solvers on GPUs, Comput. Phys. Commun. 181 (2010) 1517-1528, [arXiv:0911.3191].

[21] R. Babich, M. A. Clark, and B. Joo, Parallelizing the QUDA Library for Multi-GPU Calculations in Lattice Quantum Chromodynamics, arXiv:1011.0024. 